AUdiology

\title{
Are smartphone applications (App) useful to improve hearing?
}

\author{
Le App per smartphone possono essere utili per migliorare l'udito?
}

\begin{abstract}
Paz Martinez-Beneyto ${ }^{1,2}$, Sebastiano Franchella ${ }^{3,4}$, Fabio Alonso Rodriguez ${ }^{1}$, Rafael Navarro-Velasquez ${ }^{1}$, Miguel A. Martinez-Beneito ${ }^{5,6}$, Alessandro Martini ${ }^{3}$, Jaime Marco Algarra ${ }^{1,2}$

1 Servicio de Otorrinolaringologia, Hospital Clinico-Universitario, Valencia, Spain; ${ }^{2}$ Departamento de Cirugía, Facultad de Medicina, Universidad de Valencia, Spain; ${ }^{3}$ Otorinolaringoiatria, Dipartimento di Neuroscienze DNS, Università degli Studi di Padova, Italy; ${ }^{4}$ Dipartimento di Salute della Donna e del Bambino SDB, Università degli Studi di Padova, Italy; ${ }^{5}$ FISABIO, Fundacion para el fomento de la investigacion sanitaria y biomedica de la Comunidad Valenciana, Valencia, Spain; ${ }^{6}$ CIBER Epidemiologia y Salud Publica (CIBERESP), Madrid, Spain
\end{abstract}

\section{SUMMARY}

The objective of the study is to assess whether a smartphone application (App) designed to improve hearing can improve audiological performance in patients with normal hearing and with varying grades of hearing loss (HL). This is a multicentre prospective analytical study. We performed a battery of audiological tests consisting of pure tone audiometry (PTA) and a word recognition test (WRT) in quiet and in noise at different signal-to-noise ratio (SNR) using or not a smartphone App. Intra-subject results under both conditions were compared to determine the App's effect on hearing. A survey was also carried out to obtain data on subjective hearing experience with the App. We recruited $55 \mathrm{HL}$ patients and 13 normalhearing controls between June to December 2017. The results show that use of the App in HL patients improved WRT scores by a mean of $30.3 \%$ in quiet, $24.3 \%$ in noise $+10 \mathrm{~dB}$ SNR, and $20.8 \%$ in $+5 \mathrm{~dB}$ SNR. App use was identified as a factor that increased word recognition (odds ratio $=1.812, \mathrm{p}<0.05$ ) and $61 \%$ of subjects rated sound quality when using the App as good or excellent. The use of a smartphone hearing App improved scores in both PTA and WRT in most cases. Patients with binaural hearing impairment $<60 \%$ obtained the best results. Subjective user satisfaction was good in both conditions.

KEY WORDS: hearing aids, hearing loss, mobile applications

\section{RIASSUNTO}

L'obiettivo dello studio è quello valutare l'efficacia di un'App per smartphone creata con lo scopo di migliorare le performance uditive sia in soggetti normoudenti che in pazienti affetti da ipoacusia da lieve a severa. Si tratta di uno studio analitico multicentrico, eseguito tra giugno e dicembre 2017, che ha analizzato un campione di 68 pazienti di cui 55 ipoacusici e 13 normoudenti; a tutti i pazienti sono stati somministrati test audiologici specifici sia durante l'utilizzo della suddetta App che in assenza di ausili uditivi. Il protocollo di valutazione audiologica prevedeva l'esecuzione di un'audiometria tonale liminare e del WRT (word recognition test) sia nel silenzio che in competizione a differenti livelli di rapporto segnale/rumore; la batteria di test è stata eseguita sia in assenza di amplificazione uditiva che utilizzando l'App per smartphone. I pazienti sono stati sottoposti ad un sondaggio relativo all'esperienza uditiva con un questionario sviluppato ad hoc. I dati preliminari sono piuttosto incoraggianti in quanto dimostrano che l'utilizzo dell'App migliora le performance del WRT nei pazienti ipoacusici. Il test ha evidenziato un miglioramento medio del 30,3\% nel silenzio, del 24,3\% nel rumore con SNR (signal to noise ratio) $+10 \mathrm{~dB}$ e del $20,8 \%$ nel rumore con $S N R+5 d B$. L'utilizzo dell'app è stato identificato come un fattore che migliora il riconoscimento verbale (Odds Ratio $=1,812, p<0,05)$ e il $61 \%$ dei soggetti ha valutato come buono o eccellente la qualità del suono utilizzando l'App. L'utilizzo dell'App per smartphone ha migliorato nella maggior parte dei casi i punteggi sia all'audiometria tonale che al WRT e i pazienti con un'ipoacusia bilaterale $<60 \%$ hanno ottenuto i risultati migliori; tutti i pazienti sono stati soddisfatti dell'App durante l'utilizzo in cabina silente.

PAROLE CHIAVE: protesi acustiche, ipoacusia, applicazioni per smartphone
Received: May 20, 2019

Accepted: November 19, 2019

Published online: June 10, 2020

\section{Correspondence}

Paz Martinez-Beneyto

Hospital Clinico Universitario de Valencia, Avenida

Blasco Ibanez 17, 46010 Valencia, Spain

Tel. 961973500 . Fax 963862600

E-mail: pazmabe@gmail.com

Funding

None.

Conflict of interest

The Authors declare no conflict of interest.

How to cite this article: Martinez-Beneyto $P$ Franchella S, Alonso Rodriguez F, et al. Are smartphone applications (App) useful to improve hearing? Acta Otorhinolaryngol Ital 2020;40:304-310. https:// doi.org/10.14639/0392-100X-N0318

() Società Italiana di Otorinolaringoiatria e Chirurgia Cervico-Facciale

\section{(c) (i) $(9)$}

This is an open access article distributed in accordance with the CC-BY-NC-ND (Creative Commons Attribution-NonCommercial-NoDerivatives 4.0 International) license. The article can be used by giving appropriate credit and mentioning the license, but only for non-commercial purposes and only in the original version. For further information: https:// creativecommons.org/licenses/by-nc-nd/4.0/deed.en 


\section{Introduction}

Millions of people suffer from disabling hearing loss (HL) that requires some kind of treatment, and hearing aids are the standard recommendation. At the same time, smartphones are now fully integrated in our lives, and these high processing capacity devices can be used to run applications (Apps) with diverse functions. Some have medical uses, under the umbrella term "e-health", as healthcare practices supported by electronic processes and communication, and among these, some Apps can amplify sound with the objective of improving listening. These Apps work by receiving sound through the smartphone microphone, and then processing the signal using algorithms for sound amplification, frequency modulation, background noise elimination, etc. The resulting signal is sent through headphones to the user, who potentially receives a more intense sound with noise reduction.

Information about these Apps is of importance to hearing professionals. Are they really useful? Can we recommend them to our patients? What kind of improvement can we expect? Do they cause sound distortion? To date few studies on Apps for audiological purposes have been published, and most are related to HL screening and diagnosis ${ }^{1-5}$, with no published data about the efficacy of Apps designed to improve hearing.

The purpose of this study is to investigate the benefits to hearing provided by an App. A prospective study was designed in which a group of hearing impaired patients and normal-hearing volunteers performed a battery of hearing tests with and without use of the App to objectively quantify the possible benefits to hearing.

\section{Materials and methods}

This is a multicentre prospective analytical study, based on an intrasubject comparison and approved by the local Research Ethics Committee. The project was conducted in two tertiary referral hospitals: the Otolaryngology division of the Hospital Clinico Universitario of Valencia (Spain) and the Otolaryngology division of the University Hospital of Padua (Italy).

\section{Subjects}

We recruited adult subjects with HL treated at the two centres in the period from June to December 2017 who met the following inclusion criteria:

1. bilateral and symmetrical HL (interaural mean threshold difference $<10 \mathrm{~dB}$ );

2. HL onset $>6$ months;

3. native in the language of the Word Recognition Test (WRT);
4. > 18 years old;

5. agreed inclusion in the study by signing informed consent.

Exclusion criteria were:

1. mental disorder that could prevent a successful test outcome;

2. acute ear infection.

We recruited a second group of normal hearing adult subjects.

\section{Smartphone and earphones}

The smartphone used for the experiment was an Apple iPhone $6 \mathrm{~S}$, iOS version 10.1.1. The sound output was connected to Sony MDR-EX15LP in-ear headphones (power handling capacity: $100 \mathrm{~mW}$, impedance: $16 \Omega$ at $1 \mathrm{kHz}$, sensitivity: $100 \mathrm{~dB} / \mathrm{mW}$ and frequency response: $8-22,000 \mathrm{~Hz}$ ).

\section{Mobile application (App)}

Several Google searches with the terms [hearing aid App], [deafness App], and [hearing amplification App] were performed. The App selected was Petralex ${ }^{\circledR}$, version 1.5.3 (developer: IT For You), as it met the following criteria: easy to use, no need for training, free of charge, and available for both Android and iOS.

Although the App allows some parameter settings, the following default settings were established for all patients: volume was set to maximum, compression was turned off, amplification mode was "NAL" ${ }^{5}$, low and high frequencies were set to medium, environmental noise reduction was activated, and the main hearing profile was set to normal hearing amplification.

\section{Hearing tests}

All participants were tested in a sound-treated booth with an ISO standard calibrated audiometer and headphones. In Hospital Clinico Universitario of Valencia the Interacoustic $\mathrm{AC} 40$ audiometer was used, while in the University Hospital of Padova the Otometrics Mercury Madsen Astera was used, with the GN Otometrics OTOsuite, version 4.75.01 software. The tests were performed in both centres by the same operator using the same smartphone and earphones.

The testing sequence is represented in Figure 1. All participants underwent pure tone audiometry (PTA) and word recognition test (WRT), as follows:

- PTA with air-conduction headphones (testing the octave points from 250 to $8,000 \mathrm{~Hz}$ ) and with bone-conduction oscillator (testing the octave points from 250 to 4,000 Hz);

- Free field (FF) testing was performed under two conditions, first without aid and second using the smartphone 




Figure 1. Subject testing steps.

connected to the earphones and using the smartphone with the Petralex ${ }^{\circledR}$ App at one metre from the speaker.

The following tests were performed:

- FF PTA (testing the frequencies 250-500-1,000-2,0003,000-4,000 Hz);

- FF-WRT at $65 \mathrm{~dB}$ HL in quiet;

- FF-WRT at $65 \mathrm{~dB}$ HL in noise at + $10 \mathrm{~dB}$ SNR (signalto-noise ratio);

- FF-WRT at $65 \mathrm{~dB}$ HL in noise at $+5 \mathrm{~dB}$ SNR.

For the WRT, 10 disyllabic words in the official language of the country were delivered and correct answers expressed in percentages. Noise was a narrow-band sound stimulus delivered from the same speaker.

\section{Survey}

All participants were asked to complete a brief survey providing subjective assessment of the hearing experience after the testing. The questions were:

1. Did you notice an improvement in your hearing when using the App? Yes/No.

2. Did you notice any sound delay? Yes/No.

3. Did you feel any kind of discomfort during App use? Yes/No.

4. Did you notice annoying noises when using the App? Yes/No.

5. Rate hearing quality when using the App. (1) Very poor; (2) Poor; (3) Appropriate; (4) Good; or (5) Excellent.

\section{Database and statistics}

A database was compiled with hearing test and survey data, and other data such as age, sex, deafness type and aetiology. Several hearing parameters were calculated using the raw data from the hearing test, as follows ${ }^{6,7}$ :

- Pure tone average: average air conduction thresholds at $500-1,000-2,000-3,000 \mathrm{~Hz}$ of the PTA with headphones.

- Percentage of $\mathrm{HL}=1,5\left(\frac{0,5 \mathrm{kHz}+1 \mathrm{kHz}+2 \mathrm{kHz}+3 \mathrm{kHz}}{4}-25\right) \%$

If the value is a negative number a value of $0 \%$ is assigned so that normal-hearing subjects have $0 \%$.

- Binaural hearing impairment $(\mathrm{BHI})$ percentage $=$

$\underline{5 *(\text { percentage of HL best ear })+\text { percentage of HL worst ear }}$

App benefit was established as the difference between the results obtained using the App and the results without its use. In order to obtain positive values when the use of the App gave improved results, and negative values when the APP impaired the results, the following formulas were devised:

- benefit obtained with App in PTA: [PTA without App] [PTA with App];

- benefit obtained with App in WRT: [WRT\% with App] [WRT\% without App].

A statistical logistic regression model was performed to analyse the effect of each variable on the audiometric test results. This model considers the interaction of each variable analysed during App use to determine which factor effects are modified by its presence.

\section{Results}

68 subjects were recruited, $51 \%$ male and $49 \%$ female, average age 54 years [range, 20-84]. Based on a cutoff hearing threshold of $20 \mathrm{~dB}$ of PTA in FF, in order to consider binaural hearing, we studied 55 subjects $(80.8 \%)$ with HL and 13 subjects (19.1\%) with normal hearing. The relevant pathologies in the hearing-impaired subjects 
were: $25(45.4 \%)$ presbycusis, $18(32.7 \%)$ chronic otitis media and $12(21.8 \%)$ other ear pathologies (Ménière disease, otosclerosis, or mixed ear pathologies). Regarding hypoacusia type, $36(52.9 \%)$ patients had sensorineural HL, $10(14.7 \%)$ conductive HL, 9 (13.2\%) mixed HL and $13(19.1 \%)$ normal hearing. Table I shows the main parameters of the entire sample, normal hearing subjects and patients with HL.

BHI distribution was split into the following ranges: $\mathrm{BHI}=0 \%$ in 13 subjects; $1-20 \%$ in 29 subjects; $21-40 \%$ in 10 subjects; $41-60 \%$ in 10 subjects; and $61-83 \%$ in 6 subjects.

The different hearing test outcomes were analysed with and without App use.

Table II shows the averages of these results and the benefit obtained when the subject was using the App. A positive benefit value means that listening performance improved with App use, while a negative value means that App use provided worse results.

The benefit values varied significantly across patients. Benefit distribution for each hearing test is represented graphically in Figure 2. Calculating the percentage of subjects obtaining better results, $31 \%$ of the subjects in quiet, $43 \%$ in $+10 \mathrm{SNR}$, and $38 \%$ in +5 SNR scored $>10 \%$ higher on the WRT with the App than without; and $20 \%$ of subjects in quiet, $22 \%$ in +10 SNR, and $26 \%$ in +5 SNR scored $>20 \%$ higher when using the App. Results with the App were worse in $11 \%$ of the subjects in quiet, $5 \%$ in +10 SNR, and $7 \%$ in +5 SNR.

A statistical logistic regression model was adjusted to

Table I. Age, sex and PTA in free field (FF) for the global sample, for the normal hearing and patients with hearing loss groups.

\begin{tabular}{lccc} 
& All & Normal hearing & Patients with HL \\
$\mathrm{N}$ & 68 & 13 & 55 \\
$\begin{array}{l}\text { Age average } \\
\text { [range] }\end{array}$ & $53.6[21-84]$ & $29.1[21-57]$ & $66[21-84]$ \\
$\begin{array}{l}\text { Sex } \\
{[\text { male/female] }}\end{array}$ & $33 / 35$ & $6 / 7$ & $27 / 28$ \\
$\begin{array}{l}\text { PTA [dB] average } \\
\text { [range] }\end{array}$ & $38.7[5-79.4]$ & $13.6[5-19.3]$ & $44.6[21.8-79.4]$ \\
\hline HL: hearing loss; PTA: pure tone average in FF. &
\end{tabular}

estimate the effect of each main variable (noise, grade and type of HL), and the subject as a random effect, on hearing test results. This regression model considers the interaction of each variable with App use to determine which of these factor's effects are modified by the App.

Table III shows the odds ratio (OR) obtained in relation to the WRT result in a normal-hearing person. An $\mathrm{OR}=1$ means that the expected results are like a normalhearing subject; an OR $<1$ indicates that the probability of understanding and repeating the words of the verbal audiometry is lower than in normal-hearing condition and an $\mathrm{OR}>1$ indicates that the probability of understanding and repeating the words is higher than normal. As seen, the presence of noise, the magnitude of BHI, decreases the probability of getting the words correct in WRT, and overall, use of the App increased it $(\mathrm{OR}=1.812, \mathrm{p}<0.05)$. The statistical model provides an estimation of the WRT score in both conditions (with and without App use) for different levels of BHI. Figure 3 shows the estimation for sensorineural and conductive HL in silence and in different noise levels. In many cases, App use improved word recognition over a certain range of hearing loss. Cases with more than $10 \%$ improvement are shaded in grey.

Finally, the results of the satisfaction survey completed by the participants showed that 43 (63\%) subjects noticed an improvement in hearing, $60(88 \%)$ did not notice sound delay, $66(97 \%)$ did not feel discomfort and $63(93 \%)$ did not hear annoying noises. Self-assessment of hearing quality, represented in Figure 4, shows $61 \%$ as good or excellent.

\section{Discussion}

The use of a smartphone + App set to improve hearing is part of the "eHealth" concept ${ }^{8}$, which is defined as Information and Communication Technologies used in the Health areas of prevention, diagnosis, treatment, monitoring and management. As health professionals, we must be open to the emergence and increasing importance of eHealth Applications and device use. These new solutions have to be tested prior to their recommendation and use, however, in order to advise patients based on scientific

Table II. Average and [range] of the hearing tests with and without App in the population analysed. Benefit is the difference between the two conditions.

\begin{tabular}{lcccccc} 
& \multicolumn{3}{c}{ Normal hearing } & \multicolumn{3}{c}{ Patients with HL } \\
PTA (dB) & No App & With App & Benefit & No App & With App & Benefit \\
WRT - quiet (\%) & $13.6[5-19.3]$ & $8.26[2.5-13.7]$ & 5.3 & $44.6[21.8-79.4]$ & $38.3[15-71.2]$ \\
WRT + 10 dB SNR (\%) & 100 & $97.7[90-100]$ & -2.3 & $34.3[0-100]$ & $67.2[0-100]$ & 30,2 \\
WRT + 5 dB SNR (\%) & $97.7[90-100]$ & $99.2[90-100]$ & 1.5 & $23.1[0-100]$ & $50.4[0-100]$ & 27,3 \\
\hline
\end{tabular}

HL: hearing IoSs; PTA: pure tone average in FF; WRT: word recognition test; SNR: signal-to-noise ratio. 


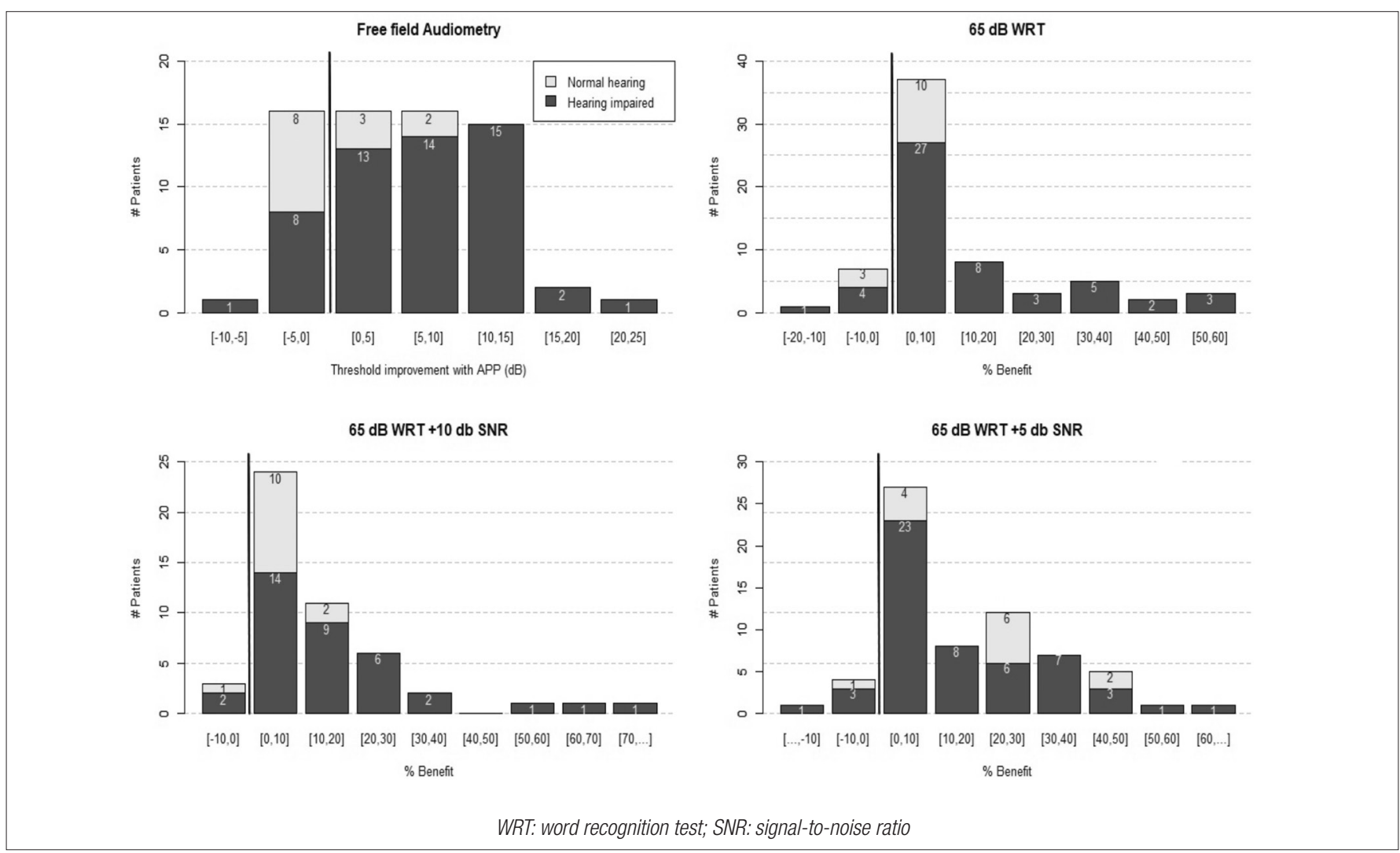

Figure 2. Distribution of benefit percentage when using the App for each hearing test. Subjects with $\mathrm{HL}$ are represented in dark grey, and normal-hearing subjects in light grey. Vertical lines split cases of no benefit from App use (left) from cases of better performance when using the App (right).

Table III. Odds ratio (OR) and p-value for each of variables analysed compared with the normal-hearing group for WRT.

\begin{tabular}{lcc} 
& OR & P value \\
Normal hearing & 1 & - \\
Conductive HL & 0.323 & 0.114 \\
Sensory neural HL & 0.129 & 0.1190 \\
Mixed HL & 0.026 & 0.0181 \\
$+5 \mathrm{~dB} \mathrm{SNR}$ & 0.065 & $<0.0001$ \\
$+10 \mathrm{~dB}$ SNR & 0.227 & $<0.0001$ \\
Binaural hearing loss & 0.880 & $<0.0001$ \\
Use of the App & 1.812 & 0.0417 \\
\hline
\end{tabular}

OR: odds ratio; HL: hearing loss; SNR: signal-to-noise ratio.

evidence. This study is the first to attempt to validate a Smartphone App as a hearing aid and objectively assess the benefits obtained in order to establish the target groups that could make use of this technology. Though the use of smartphone-connected hearing aid is increasing due to the wide possibility of customising hearing aid amplification in different situations ${ }^{9,10}$, few studies consider its use specifically for hearing improvement. Moreover, these studies just consider the users' satisfaction without taking into account the auditory gain ${ }^{11}$.
Herein, we observed a moderate improvement in tonal audiometry thresholds (average $6.2 \mathrm{~dB}$ ) in the $\mathrm{HL}$ population. However, a remarkable gain in WRT was obtained both in silence and with competing noise at $+10 \mathrm{~dB}$ and $+5 \mathrm{~dB}$ SNR, showing a word recognition improvement of $30 \%, 24 \%$ and $20 \%$, respectively. The results in normal-hearing subjects were lower, between $0.8 \%$ and $8.4 \%$ for WRT, which can be attributed in part to the ceiling effect of the test we used, since the near $100 \%$ basal levels in this population were difficult to improve on. The logistic regression statistical model convincingly demonstrates that use of the App improves word recognition $(\mathrm{OR}=1.812, \mathrm{p}<0.05)$ regardless of its interaction with any other variable.

Testing different patients in different centres with different audiometers and test material could be considered a bias, but a negligible one due to the fact that each subject acts as their own control, as all comparisons are intra-subject.

In line with the predictions of the statistical model, and choosing $>10 \%$ improvement in WRT results as an arbitrary and acceptable benefit with the App, we observed that in silence, this benefit could be obtained in cases of $\mathrm{BHI}<55 \%$ for conductive $\mathrm{HL}$, and $<60 \%$ for sensorineural 


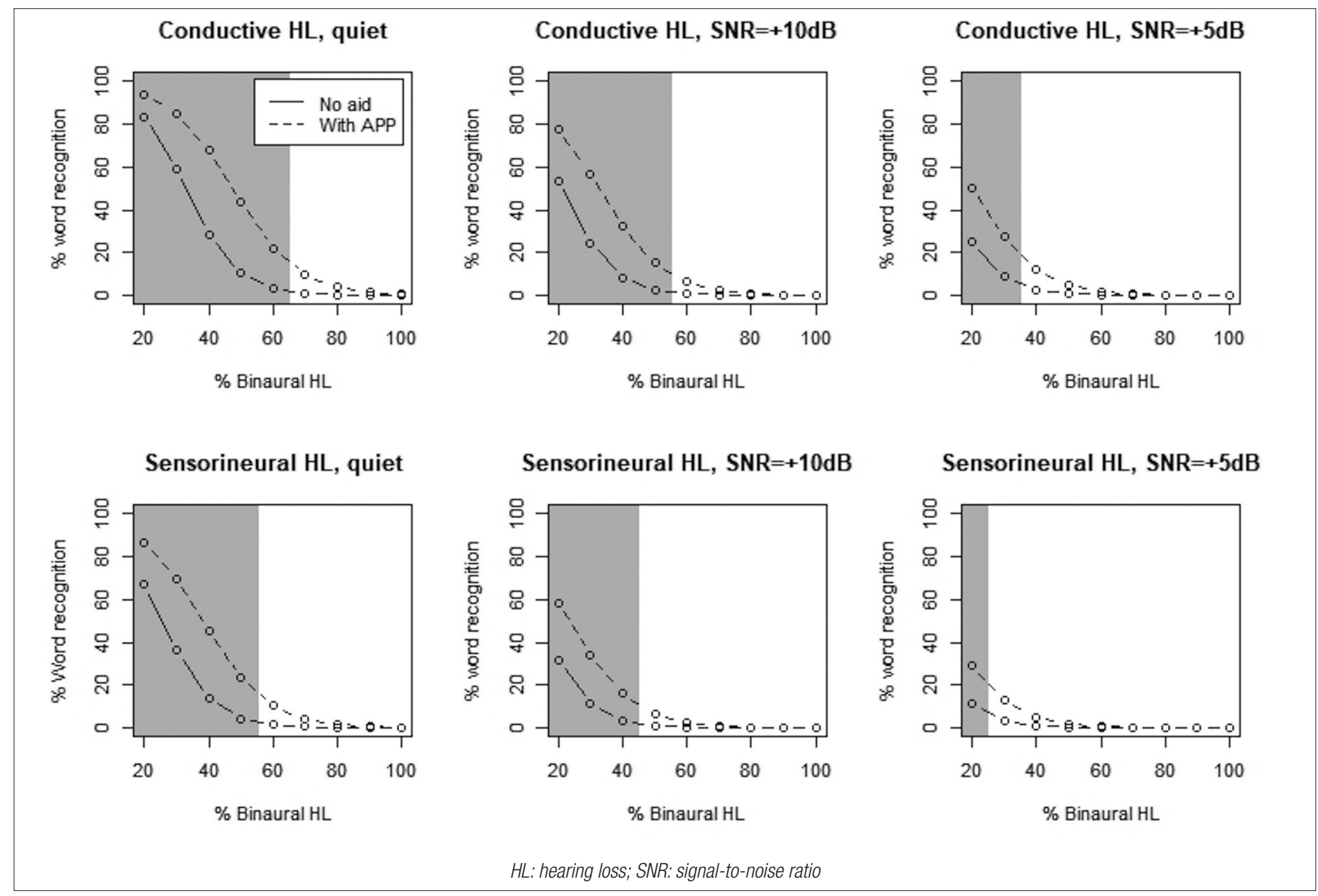

Figure 3. Prediction of verbal comprehension with no aid (solid lines), and using the App (dashed lines). The range of binaural loss with > 10\% of improvement in WRT is shaded in grey.

HL, a potential limit for recommending the App to obtain improvement. Hearing under noise conditions are more challenging and the BHI to get $>10 \%$ in WRT improvement is logically lower.



Figure 4. Self-assessment of hearing quality. Answers to the question "Rate hearing quality when using the App".
Admittedly, some participants obtained worse results with the use of the App, which should be taken into account, although they were not significantly worse $(<10 \%$ for WRT), which can be explained by the distortion effect that the device can have on natural sound, or by poor headset placement. However, participants gave very good evaluations in the questionnaire, expressing minimal perception of discomfort or annoying noises and a high level of overall satisfaction.

In light of our results, use of this App provides some benefit in most cases, especially under silence conditions, for patients suffering mild and medium hearing loss (audiometric thresholds $<60 \mathrm{~dB}$ ). The benefit is lower with the presence of background noise and poorer audiometric values.

Beyond purely audiometric aspects, other factors, such as ergonomics or ease of use, are also of importance in mobile phone use for sound amplification. Although this study has not addressed these aspects, it seems obvious that conventional hearing aids are more user-friendly. Using 
the App as a hearing aid means: 1) wearing the earphones continuously, although wireless models could be more wearable; 2) holding the smartphone rather than carrying it in the pocket, if the earphones do not include a built-in microphone; and 3) possibly preventing the simultaneous use of other tasks on the device. Issues in everyday use define the Smartphone + App set as a rather cumbersome hearing aid, implying that its use could be limited to specific situations such as meetings, conferences, watching $\mathrm{TV}$, etc... rather than habitual use. Nonetheless, an additional advantage of the Smartphone + App set is the possibility of focusing the microphone and moving it closer to the sound source, thus improving the SNR when listening in noisy environments. Another advantage is the fact that mobile phones are widely distribuited, also in the elderly population, for whom several studies have found association between aided hearing outcomes and cognitive skills ${ }^{12}$.

The present study breaks new ground in analysing the use of a smartphone App as a hearing aid, with demonstrated benefits. However, some limitations should be pointed out as proposals for future research. This research was carried out with a specific set of headphones, a single App (of the many available on the market) and one Smartphone device, to provide homogeneity to the sample. In addition, for this study we used only one amplification profile, which amplified all frequencies equally, while the app has the possibility to amplify the different frequencies according to the needs of the patient. However, more research is needed using other software and hardware combinations, which should be taken into account when interpreting our results. Additionally, future system testing should be extended beyond the audiometric booth to real-life situations. Another limitation of the study is that we did not measure the output of the device when using the App, so that the actual amplification could not be estimated. However, this evaluation was not part of our study because our goal was to assess the effectiveness of this product in order to offer advice to our patients.

Nevertheless, this first approach indicates a promising future for smartphones as a hearing aid in particular hearing impairments and circumstances.

\section{Conclusions}

In most cases, use of a smartphone hearing App provides better scores in both PTA and WRT. Greatest benefit is obtained by patients with $\mathrm{BHI}<60 \%$. App use in background noise situations improves performance, although the benefit is lesser. Subjective user satisfaction was good in booth conditions.

\section{Acknowledgements}

We thank the other members of the ENT teams for their help in assisting patients.

\section{References}

1 Maidment DW, Barker AB, Xia J, et al. Effectiveness of alternative listening devices to conventional hearing aids for adults with hearing loss: a systematic review protocol. BMJ Open 2016;6:e011683. https://doi.org/10.1136/bmjopen-2016-011683

2 Amlani A. Improving patient compliance to hearing healthcare services and treatment through self-efficacy and smartphone applications. Hearing Review 2015;21:16.

3 Amlani AB, Taylor C, Levy R, et al. Utility of smartphone-based hearing aid Applications as a substitute to traditional hearing aids. Hearing Review 2103;19:17.

4 Derin S, Cam OH, Beydilli H, et al. Initial assessment of hearing loss using a mobile Application for audiological evaluation. J Laryngol Otol 2016;130:248-51. https://doi.org/10.1017/S0022215116000062

5 Byrne D, Tonisson W. Selecting the gain of hearing aids for persons with sensorineural hearing impairments. Scand Audiol 1976;5:51-9. https://doi.org/10.3109/01050397609043095

6 Real Decreto 1971/1999, de 23 de diciembre, de procedimiento para el reconocimiento, declaración y calificación del grado de minusvalía. Boletín Oficial del Estado n. 22, 26 de Enero de 2000, 3317-90.

7 Manrique RM, Algarra MJ. Audiología. Madrid: CYAN, Proyectos Editoriales, S.A.; 2014.

8 Oh H, Rizo C, Enkin M, at al. What is eHealth: a systematic review of published definitions. J Med Internet Res 2005;7:e1. https://doi. org/10.2196/jmir.7.1.e1

9 Aberdeen L, Fereiro D. Communicating with assistive listening devices and age-related hearing loss: perceptions of older Australians. Contemp Nurse 2014;47:119-31. https://doi.org/10.5172/ conu.2014.47.1-2.119

10 Lopez EA, Costa OA, Ferrari DV. Development and technical validation of the mobile based assistive listening system: a smartphonebased remote microphone. Am J Audiol 2016;25:288-94. https://doi. org/10.1044/2016_AJA-16-0016

11 Maidment DW, Ferguson M. An application of the medical research council's guidelines for evaluating complex interventions: a usability study assessing smartphone-connected listening devices in adults with hearing loss. Am J Audiol 2018;27:474-81. https://doi. org/10.1044/2018_AJA-IMIA3-18-0019

12 Tognola G, Mainardi A, Vincenti V, et al. Benefit of hearing aid use in the elderly: the impact of age, cognition and hearing impairment. Acta Otorhinolaryngol Ital 2019;39:409-418. https://doi. org/10.14639/0392-100X-2165 